\title{
El neoliberalismo y los cambios en la elite de poder en México
}

\author{
Larissa Adler- Lomnitz \\ Jorge Gil-Mendieta \\ Instituto de Investigaciones en Matemáticas Aplicadas y en Sistemas \\ Universidad Nacional Autónoma de México
}

\section{I ntroducción}

A partir de la revolución de 1910, el Estado mexicano creó y reguló el sistema político que gobernaría al país durante el siglo XX. Concluido el periodo de los presidentes militares (1940) la mayoría de los altos cargos del aparato estatal en México fueron ocupados por abogados entrenados en la Universidad Nacional Autónoma de México donde adquirían el conocimiento formal de la ley y aprendían a desarrollar habilidades relacionadas con el quehacer político en México y la construcción de nuevas redes sociales.

En la década de los años ochentas, el país sufrió una crisis económica conocida como la "crisis de la deuda externa". Como resultado, las administraciones públicas que siguieron vieron la necesidad de introducir ajustes estructurales basados en el "Consenso de Washington" que promovía la implementación del modelo neoliberal. Este modelo en vez de enfatizar la estabilidad política y un desarrollo económico proteccionista y de bienestar social, promovía la economía de mercado, la desregulación de los mercados eliminando las barreras para el comercio internacional, la reducción del gasto público y por ende la disminución del aparato estatal, como puede verse en la Figura 2. En otras palabras, México entraba al sistema global. Como consecuencia, una nueva elite de tecnócratas comenzó a ocupar los altos cargos del gobierno. Estos nuevos profesionales (economistas de formación monetarista y abogados especializados en asuntos internacionales) provienen de familias de clase media alta o alta, con educación cosmopolita y estudian en universidades privadas de elite. Generalmente pasan un periodo de entrenamiento especializado en universidades del extranjero en donde establecen nuevas redes sociales globales con profesores norteamericanos y estudiantes latinos y mexicanos que pueden, eventualmente, ocupar posiciones similares en la elite de tecnócratas de su país o en organizaciones supranacionales como el Banco Mundial o el Fondo Monetario Internacional. Estas relaciones les permitirán desenvolverse en el mundo de las finanzas y el comercio internacional y así tener un conocimiento mayor de cómo introducir al país en el mundo globalizado de hoy.

En este análisis utilizamos la Base de Datos REDMEX II, 2001. Laboratorio de Redes IIMASUNAM, reportada por Jorge Gil- Mendieta et al en el SUUNBELT XXI International Conference on Social Networks, Budapest, Hungría, Abril de 2001. Esta base contiene las biografías de 6,000 individuos activos en política, de ahí se extrajo la información sobre los abogados y economistas que se presenta en las tablas y gráficas del Anexo 1.

En la Tabla 1 se presenta el número de abogados, economistas y otros profesionales desde 1940 hasta 1993, se analizó la base de datos completa. En la Tabla 2 se presentan datos porcentuales de abogados y economistas entre 1940 y 1993. En la Figura 3 se comparan porcentualmente abogados y economistas a lo largo de nueve periodos presidenciales.

En este análisis se muestra el aumento progresivo de los economistas en los niveles altos del aparato estatal, así como la evolución de las redes sociales de algunos de estos personajes. De particular interés son los casos de Ernesto Zedillo, anterior presidente de México y primer economista que ha llegado a la Presidencia y el de Francisco Gil, subsecretario de Hacienda en el gobierno de Salinas (1988-1994) y Secretario de Hacienda 
en el gabinete del actual Presidente Vicente Fox. Presentaremos un análisis de las tendencias de los números de abogados y economistas a través del tiempo.

En términos del sistema político mexicano la sucesión consecutiva de tres presidentes que aplicaron las mismas políticas de corte neoliberal, excluyendo a los círculos tradicionales del partido político implicó que durante dieciocho años no se aplicó el efecto llamado de "péndulo". Los tres últimos presidentes compartieron las mismas redes compuestas de economistas y técnicos entrenados en Estados Unidos, así como una tendencia opuesta a la del desarrollismo post-revolucionario. Su ideología se conforma a una nueva modernidad globalizante basada en una diferente conceptualización del papel del Estado y del mercado. Es relevante el hecho de que los tres últimos presidentes del PRI surgieron de la ahora desaparecida Secretaria de Programación y Presupuesto. De la Madrid y Salinas se encargaron de dar un lugar preponderante en sus equipos de trabajo a los funcionarios ligados a esa Secretaría (Camp, 1996: 307), dándole, por lo tanto, mayor énfasis a secretarias relacionadas con la economía que a la anteriormente más poderosa Secretaría de Gobernación encargada tradicionalmente del control político del Estado. Los cambios impuestos por las condiciones económicas y que corresponden a la introducción de la globalización y del neoliberalismo, se hicieron a través de una nueva élite tecnocrática que, a su vez, se mantuvo gracias al sistema tradicional del clientelismo, redes sociales y camarillas. Podemos observar en las Tablas 5,6 y 7, correspondientes a los gobiernos de Miguel De La Madrid, Carlos Salinas de Gortari y Ernesto Zedillo que el porcentaje de abogados en el gabinete pasó de $36 \%$ a $29 \%$ y en el sexenio de Zedillo a $32 \%$ y, el porcentaje de economistas en el gabinete de De la Madrid fue de $27 \%$, en el de Salinas $39 \%$ y en el de Zedillo $45 \%$. Habiendo también compartido diferentes funcionarios en estos tres gobiernos.

Aparece pues, un pequeño grupo de tecnócratas entrenados en Estados Unidos que pudieron comunicarse con las redes internacionales dominantes en la nueva etapa de la globalización (Centeno, 1994: 37, 96.). En estas décadas, si bien se trató de reducir el aparato estatal, se produjo una mayor centralización del poder de la burocracia dentro del Ejecutivo, con un aumento de la importancia del sector de planificación financiera. Con respecto a esto, una especialista en el caso de México señala:

\begin{abstract}
"Un desarrollo crucial fue el que esta élite cohesionada de personas especializadas con la capacidad de aplicar instrumentos racionales técnicos dominaron el Estado. Los cambios que se fueron produciendo habrían sido imposibles sin que la burocracia fuera dominada por un grupo de tecnocratas que alcanzó puestos en el gobierno a través de canales burocráticos en vez de canales electorales y corporativos. Así se fue dando un declive de la élite tradicional política. Esta nueva élite... no solamente mantenía el poder detrás del trono, sino que empezó a comandar el Estado." (Centeno, 1994: 40).
\end{abstract}

Haciendo un análisis de las redes de los 85 funcionarios: abogados y economistas, de alto nivel que participaron en los gabinetes de De la Madrid, Salinas y Zedillo, a partir de 1940 hasta 1993, observamos el mismo comportamiento que en el caso general. Tablas 3 y 4. Figuras 4, 5 y 6. En particular en la Figura 6, podemos notar la tendencia creciente del porcentaje de economistas contra abogados.

El surgimiento de esta nueva élite no habría sido posible sin el apoyo tradicional del sistema complejo de redes y de relaciones patrón-cliente (ver Lomnitz, 1987). Las redes tradicionales aseguraron que la nueva élite subiera al poder, controlara los recursos y también produjera los cambios del nuevo proceso. Asimismo, desde el punto de vista formal, la desregulación en materia económica no vino acompañada de una disminución de 
las capacidades de intervención del poder Ejecutivo sobre la vida económica. La Constitución mexicana faculta al presidente para legislar en materia de comercio exterior, para imponer aranceles a las exportaciones y a las importaciones y para fijar precios y prioridades de producción, además de que consagra en el artículo 28 la rectoría económica del Estado (CIDAC, 1994: 29).

El aparente triunfo de la nueva política económica tuvo también un costo político para el partido en el poder (PRI). Por un lado, la suspensión del "péndulo", con la consiguiente exclusión de los grupos no relacionados con la élite tecnocrática, provocó una escisión que después daría lugar al Partido de la Revolución Democrática (PRD), el partido opositor de centro-izquierda. Por otra parte, la implementación de las políticas de ajuste tuvo fuertes costos sociales que se reflejaron en un descontento expresado en el proceso electoral (Przeworski, 1995: cap. 4). Esto explica el fortalecimiento tanto del PRD como del Partido de Acción Nacional (PAN), partido de centro-derecha que, aunque se mantuvo permanentemente como partido de oposición, fue solamente en los últimos años que pudo disputar la hegemonía en el poder y por primera vez ganar las elecciones presidenciales del 2000 con la victoria de Vicente Fox.

En lo que se refiere a las élites estatales concentradas en la presidencia, se ha notado también un cambio socio-cultural. El énfasis que se le da a las políticas económicas, a la internacionalización del comercio, a la dependencia hacia un mundo financiero internacional, en fin, la inserción de México en la economía global ha requerido de personas con una preparación técnica especializada ("los tecnócratas"), menos preparados para el control político de la sociedad. La mayor importancia que se le da a la economía ha implicado que, como profesionistas, economistas de formación monetarista educados en instituciones privadas ligadas con el sector financiero, han ido reemplazando a los abogados tradicionales en las posiciones privilegiadas de la élite gubernamental, Tabla 3 y 4. Figuras 4, 5 y 6. Babb (1997), en un estudio que aborda la introducción de modelos económicos internacionales en México, se centra en el caso de una élite de economistas entrenados en el extranjero que ha llegado a constituir una pequeña pero poderosa red que introdujo las nuevas políticas "modernas" de la globalización.

En un estudio de caso reciente sobre la práctica de la profesión de abogados en México (Lomnitz y Salazar, 2001) encontramos que durante 50 años o más, la mayor proporción de los abogados del país fueron entrenados en la Universidad Nacional de México siguiendo la ideología nacionalista posrevolucionaria del partido en el poder. La intención del régimen era apoyar el proyecto nacional de industrialización centrado en las ciudades, promoviendo la movilidad social y la creación y fortalecimiento de una nueva clase media a través de un esfuerzo educacional y de la creación de empleos de clase media tanto en la industria como en el aparato estatal. Asimismo, encontramos que una proporción importante de abogados trabajaba en todos los niveles del sector público, incluyendo en forma destacada, la cúspide de la élite del poder. A partir de 1946 y hasta 1988, todos los presidentes fueron abogados, y desde 1940 los gabinetes presidenciales también estaban dominados por abogados. Es sólo a partir de la administración del presidente Echeverría (1970-1976) que comienza a aumentar el número de economistas en los altos mandos, llegando al máximo porcentual en la administración del presidente Zedillo (1994-2000). Figuras 2 y 3.

Otro de los aspectos destacados del estudio se refiere a la importancia de las redes sociales en la práctica de la profesión. Mostramos que el éxito de un abogado depende no solamente de su conocimiento formal de la ley, sino también de su habilidad "informal" para construir nuevas redes sociales. Estas redes son indispensables para la colocación del profesional en el mercado laboral y también para su práctica profesional exitosa. El hecho de ser entrenado en la Universidad Nacional permitía al estudiante relacionarse no sólo con 
sus compañeros sino también con sus profesores, quienes los reclutaban para laborar tanto en el sector público como en el privado.

La "nueva modernidad" ha tenido como consecuencia el surgimiento de una nueva élite tecnocrática y una elite de abogados especializados en temas de la economía global. Estos nuevos especialistas tienen un origen socio-económico diferente al del estudiante medio de la UNAM, el cual, en su mayoría, representa a una clase media y media baja. El hecho de compartir el curriculum y la vida estudiantil durante tantos años, reforzaba una cierta cultura política dominante, muy ligada tanto al PRI como al aparato estatal y con un carácter nacionalista. En contraste, la nueva élite tecnocrática de los abogados proviene de familias de clase media alta o alta, con educación cosmopolita (importantemente saben inglés) y estudian en dos o tres universidades privadas de élite. En estas universidades adquieren, además del conocimiento tradicional de las normas constitucionales, un expertise técnico-especializado en áreas como comercio internacional, inversiones, contratos, finanzas, prácticas y costumbres de otros países, etc. En otras palabras, estos estudiantes provienen del sector privado y se preparan para trabajar en él. Curiosamente, después de pasar un período de entrenamiento especializado en universidades privadas en el extranjero, estos estudiantes regresan a ser parte de la élite tecnocrática del Estado, ubicándose principalmente en los departamentos jurídicos de las dependencias financieras, aunque, en muchos casos regresan a formar sus propios despachos privados.

Esta élite considera que el éxito en su práctica profesional no dependía de sus redes sociales. Sin embargo, en la realidad continúan usando otro tipo de redes sociales para obtener sus posiciones y el éxito. Estas redes no son las que tradicionalmente se conformaban entre estudiantes y profesores en México, sino que son establecidas en el extranjero entre estudiantes mexicanos, latinoamericanos y de otros países, entrenados en universidades norteamericanas, así como con los profesores y estudiantes norteamericanos que en el futuro pueden convertirse en funcionarios de las instituciones financieras internacionales. Estas redes ya tienen un carácter global que les permitirá desenvolverse en el mundo de las finanzas y el comercio internacional y eventualmente ocupar cargos en la élite del gobierno de sus países.

En resumen, la operación del sistema político mexicano, en la que una serie de reglas informales y características culturales han dado al régimen un carácter distinto al prescrito por la ley, ha venido sufriendo cambios como consecuencia de la globalización. La apertura económica y política, la globalización de los medios de comunicación y la influencia que ésta ha tenido en la política interna del país, han producido un cambio en la estructura de poder y su cultura política. Sobretodo en las redes sociales de la élite política compuesta mayoritariamente por tecnócratas (economistas y otros especialistas en derecho internacional) de niveles socio-económicos altos, entrenados en universidades privadas que continúan sus estudios en universidades norteamericanas en donde se especializan en temas de importancia global, aprenden nuevas teorías económicas, establecen relaciones cercanas con estudiantes y profesores que mantienen contacto con las instituciones internacionales, lo que en el futuro les permite crear redes globales y eventualmente, ocupar cargos en la élite gubernamental de sus respectivos países o en las instituciones supranacionales (Banco Mundial y/o Fondo Monetario Internacional). 


\section{Bibliografía}

Babb, Sarah (n.d.): “Money Doctors, International Constituencies, and the Legitimization of Expert Knowledge: Mexican Economics", 1929-present: documento presentado en: American Sociological Association meeting, August, mimeo.

Bresser Pereira, Luiz Carlos (1993): “Reformas Económicas y Crecimiento Económico: Eficiencia y Política en América Latina", en: Luis Carlos Bresser Pereira, J osé Maria Maravall y Adma Przeworski, Las Reformas Económicas en las Nuevas Democracias. Un Enfoque Socialdemócrata, Alianza, Madrid. Pgs. 27-100.

Camp, Roderic (1996): Reclutamiento Político en México, S. XXI, México.

Centeno, Miguel Angel (1994): Democracy within Reason. Technocratic Revolution in Mexico, The Pennsylvania State University Press, Pennsylvania.

Castañeda, Jorge (1999): La Herencia. Arqueología de la sucesión presidencial, Siglo XXI, México.

Gil- Mendieta, Jorge y Lomnitz Adler Larissa (1999), “Networks of lawyers and economists and the introduction of the new liberal policies in Mexico". Conferencia Internacional y $\mathrm{V}$ Europea de Análisis de Redes Sociales. Universitat de Barcelona, Networks Analysis Group y Universidad Autónoma de Barcelona. 28 al 31 de mayo.

Lomnitz Adler, Larissa y Rodrigo Salazar (2001): "Cultural Elements in the Practice of Law in Mexico", en Dezalay Yves and Bryiant Garth, New Challenges for the Rule of Law, American Bar Foundation, en vías de publicación.

Lomnitz Adler, Larissa (1987), "Las relaciones horizontales y verticales en la estructura social urbana

de México" en: La heterodoxia recuperada. En torno a Angel Palerm, S. Glanz Ed. México: Fondo de

Cultura Económica 
Tabla 1. Funcionarios Públicos abogados, economistas y de otras profesiones 19401993.

\section{(Base de Datos Completa)}

\begin{tabular}{|c|c|c|c|c|}
\hline Años & Abogados & Economistas & Otros & Total \\
\hline 1940 & 14 & 1 & 11 & 26 \\
\hline 1941 & 20 & 3 & 12 & 35 \\
\hline 1942 & 22 & 3 & 20 & 45 \\
\hline 1943 & 28 & 6 & 17 & 51 \\
\hline 1944 & 39 & 7 & 24 & 70 \\
\hline 1945 & 42 & 8 & 31 & 81 \\
\hline 1946 & 54 & 8 & 36 & 98 \\
\hline 1947 & 63 & 9 & 39 & 111 \\
\hline 1948 & 72 & 10 & 49 & 131 \\
\hline 1949 & 81 & 12 & 50 & 143 \\
\hline 1950 & 92 & 11 & 69 & 172 \\
\hline 1951 & 106 & 12 & 76 & 194 \\
\hline 1952 & 117 & 16 & 86 & 219 \\
\hline 1953 & 130 & 25 & 98 & 253 \\
\hline 1954 & 141 & 29 & 122 & 292 \\
\hline 1955 & 167 & 33 & 137 & 337 \\
\hline 1956 & 183 & 40 & 149 & 372 \\
\hline 1957 & 202 & 46 & 168 & 416 \\
\hline 1958 & 217 & 57 & 186 & 460 \\
\hline 1959 & 260 & 63 & 216 & 539 \\
\hline 1960 & 296 & 77 & 259 & 632 \\
\hline 1961 & 321 & 92 & 277 & 690 \\
\hline 1962 & 353 & 103 & 316 & 772 \\
\hline 1963 & 386 & 102 & 355 & 843 \\
\hline 1964 & 443 & 117 & 398 & 958 \\
\hline 1965 & 470 & 132 & 470 & 1072 \\
\hline 1966 & 503 & 146 & 496 & 1145 \\
\hline 1967 & 531 & 171 & 553 & 1255 \\
\hline 1968 & 573 & 199 & 591 & 1363 \\
\hline 1969 & 609 & 211 & 619 & 1439 \\
\hline 1970 & 726 & 252 & 721 & 1699 \\
\hline 1971 & 796 & 288 & 803 & 1887 \\
\hline 1972 & 837 & 327 & 869 & 2033 \\
\hline 1973 & 904 & 352 & 952 & 2208 \\
\hline 1974 & 933 & 382 & 1017 & 2332 \\
\hline 1975 & 996 & 406 & 1066 & 2468 \\
\hline 1976 & 1058 & 430 & 1162 & 2650 \\
\hline 1977 & 1045 & 484 & 1203 & 2732 \\
\hline 1978 & 1079 & 509 & 1264 & 2852 \\
\hline 1979 & 1132 & 539 & 1309 & 2980 \\
\hline 1980 & 1112 & 534 & 1341 & 2987 \\
\hline 1981 & 1111 & 526 & 1368 & 3005 \\
\hline 1982 & 1079 & 532 & 1395 & 3006 \\
\hline 1983 & 989 & 499 & 1297 & 2785 \\
\hline
\end{tabular}




\begin{tabular}{|c|c|c|c|c|}
\hline 1984 & 938 & 475 & 1196 & 2609 \\
\hline 1985 & 927 & 474 & 1153 & 2554 \\
\hline 1986 & 852 & 430 & 1044 & 2326 \\
\hline 1987 & 773 & 415 & 961 & 2149 \\
\hline 1988 & 737 & 408 & 913 & 2058 \\
\hline 1989 & 750 & 430 & 990 & 2170 \\
\hline 1990 & 626 & 329 & 789 & 1744 \\
\hline 1991 & 652 & 346 & 855 & 1853 \\
\hline 1992 & 649 & 345 & 875 & 1869 \\
\hline 1993 & 684 & 354 & 956 & 1994 \\
\hline
\end{tabular}

Fuente: Jorge Gil-Mendieta, S. Schmidt, J. Castro y A. Ruiz. Base de Datos REDMEX_II, 2001. Laboratorio de Redes II MAS-UNAM. 
Tabla 2. Funcionarios Públicos abogados y economistas 1940-1993. (Porcentaje como parte del total de economistas y abogados)

\begin{tabular}{|c|c|c|}
\hline Años & Abogados(\%) & Economistas(\%) \\
\hline 1940 & 93.33 & 6.67 \\
\hline 1941 & 86.96 & 13.04 \\
\hline 1942 & 88.00 & 12.00 \\
\hline 1943 & 82.35 & 17.65 \\
\hline 1944 & 84.78 & 15.22 \\
\hline 1945 & 84.00 & 16.00 \\
\hline 1946 & 87.10 & 12.90 \\
\hline 1947 & 87.50 & 12.50 \\
\hline 1948 & 87.80 & 12.20 \\
\hline 1949 & 87.10 & 12.90 \\
\hline 1950 & 89.32 & 10.68 \\
\hline 1951 & 89.83 & 10.17 \\
\hline 1952 & 87.97 & 12.03 \\
\hline 1953 & 83.87 & 16.13 \\
\hline 1954 & 82.94 & 17.06 \\
\hline 1955 & 83.50 & 16.50 \\
\hline 1956 & 82.06 & 17.94 \\
\hline 1957 & 81.45 & 18.55 \\
\hline 1958 & 79.20 & 20.80 \\
\hline 1959 & 80.50 & 19.50 \\
\hline 1960 & 79.36 & 20.64 \\
\hline 1961 & 77.72 & 22.28 \\
\hline 1962 & 77.41 & 22.59 \\
\hline 1963 & 79.10 & 20.90 \\
\hline 1964 & 79.11 & 20.89 \\
\hline 1965 & 78.07 & 21.93 \\
\hline 1966 & 77.50 & 22.50 \\
\hline 1967 & 75.64 & 24.36 \\
\hline 1968 & 74.22 & 25.78 \\
\hline 1969 & 74.27 & 25.73 \\
\hline 1970 & 74.23 & 25.77 \\
\hline 1971 & 73.43 & 26.57 \\
\hline 1972 & 71.91 & 28.09 \\
\hline 1973 & 71.97 & 28.03 \\
\hline 1974 & 70.95 & 29.05 \\
\hline 1975 & 71.04 & 28.96 \\
\hline 1976 & 71.10 & 28.90 \\
\hline 1977 & 68.35 & 31.65 \\
\hline 1978 & 67.95 & 32.05 \\
\hline 1979 & 67.74 & 32.26 \\
\hline 1980 & 67.56 & 32.44 \\
\hline 1981 & 67.87 & 32.13 \\
\hline 1982 & 66.98 & 33.02 \\
\hline 1983 & 66.47 & 33.53 \\
\hline 1984 & 66.38 & 33.62 \\
\hline 1985 & 66.17 & 33.83 \\
\hline 1986 & 66.46 & 33.54 \\
\hline
\end{tabular}




\begin{tabular}{|c|c|c|}
\hline 1987 & 65.07 & 34.93 \\
\hline 1988 & 64.37 & 35.63 \\
\hline 1989 & 63.56 & 36.44 \\
\hline 1990 & 65.55 & 34.45 \\
\hline 1991 & 65.33 & 34.67 \\
\hline 1992 & 65.29 & 34.71 \\
\hline 1993 & 65.90 & 34.10 \\
\hline
\end{tabular}

Fuente: Jorge Gil-Mendieta, S. Schmidt, J. Castro y A. Ruiz. Base de Datos REDMEX_II, 2001. Laboratorio de Redes IIMAS-UNAM. 
Tabla 3. Funcionarios Públicos abogados, economistas y otras profesiones 19401993.

( 85 personas de los gabinetes de De La Madrid, Salinas y Zedillo)

\begin{tabular}{|c|c|c|c|c|}
\hline Años & Abogados & Economistas & Otros & Total \\
\hline 1940 & 11 & 0 & 7 & 18 \\
\hline 1941 & 15 & 2 & 6 & 23 \\
\hline 1942 & 16 & 2 & 9 & 27 \\
\hline 1943 & 19 & 5 & 10 & 34 \\
\hline 1944 & 30 & 6 & 12 & 48 \\
\hline 1945 & 33 & 5 & 17 & 55 \\
\hline 1946 & 41 & 5 & 19 & 65 \\
\hline 1947 & 52 & 6 & 21 & 79 \\
\hline 1948 & 58 & 8 & 27 & 93 \\
\hline 1949 & 63 & 10 & 29 & 102 \\
\hline 1950 & 69 & 9 & 42 & 120 \\
\hline 1951 & 81 & 10 & 44 & 135 \\
\hline 1952 & 85 & 13 & 56 & 154 \\
\hline 1953 & 99 & 21 & 65 & 185 \\
\hline 1954 & 101 & 25 & 76 & 202 \\
\hline 1955 & 121 & 29 & 89 & 239 \\
\hline 1956 & 136 & 36 & 90 & 262 \\
\hline 1957 & 153 & 41 & 111 & 305 \\
\hline 1958 & 164 & 50 & 117 & 331 \\
\hline 1959 & 201 & 57 & 144 & 402 \\
\hline 1960 & 227 & 69 & 174 & 470 \\
\hline 1961 & 246 & 84 & 187 & 517 \\
\hline 1962 & 273 & 95 & 212 & 580 \\
\hline 1963 & 295 & 93 & 237 & 625 \\
\hline 1964 & 323 & 105 & 267 & 695 \\
\hline 1965 & 334 & 119 & 316 & 769 \\
\hline 1966 & 363 & 130 & 338 & 831 \\
\hline 1967 & 382 & 152 & 371 & 905 \\
\hline 1968 & 397 & 175 & 395 & 967 \\
\hline 1969 & 420 & 183 & 412 & 1015 \\
\hline 1970 & 502 & 219 & 471 & 1192 \\
\hline 1971 & 568 & 247 & 522 & 1337 \\
\hline 1972 & 594 & 281 & 557 & 1432 \\
\hline 1973 & 637 & 305 & 601 & 1543 \\
\hline 1974 & 649 & 332 & 636 & 1617 \\
\hline 1975 & 689 & 347 & 668 & 1704 \\
\hline 1976 & 739 & 373 & 732 & 1844 \\
\hline 1977 & 728 & 415 & 762 & 1905 \\
\hline 1978 & 748 & 437 & 794 & 1979 \\
\hline 1979 & 775 & 460 & 816 & 2051 \\
\hline 1980 & 747 & 456 & 827 & 2030 \\
\hline 1981 & 740 & 450 & 846 & 2036 \\
\hline 1982 & 721 & 459 & 852 & 2032 \\
\hline 1983 & 638 & 420 & 793 & 1851 \\
\hline
\end{tabular}




\begin{tabular}{|l|l|l|l|l|}
\hline 1984 & 596 & 393 & 720 & 1709 \\
\hline 1985 & 584 & 389 & 684 & 1657 \\
\hline 1986 & 518 & 349 & 603 & 1470 \\
\hline 1987 & 471 & 331 & 539 & 1341 \\
\hline 1988 & 464 & 329 & 510 & 1303 \\
\hline 1989 & 462 & 348 & 541 & 1351 \\
\hline 1990 & 355 & 257 & 395 & 1007 \\
\hline 1991 & 372 & 266 & 419 & 1057 \\
\hline 1992 & 362 & 264 & 417 & 1043 \\
\hline 1993 & 360 & 260 & 417 & 1037
\end{tabular}

Fuente: Jorge Gil-Mendieta, S. Schmidt, J. Castro y A. Ruiz. Base de Datos REDMEX_II, 2001. Laboratorio de Redes IIMAS - UNAM. 
Tabla 4. Funcionarios Públicos abogados y economistas (Porcentaje como parte del total de economistas y abogados)

( 85 personas de los gabinetes de De La Madrid, Salinas y Zedillo)

\begin{tabular}{|c|c|c|}
\hline Años & Abogados (\%) & Economistas (\%) \\
\hline 1940 & 100.00 & 0.00 \\
\hline 1941 & 88.24 & 11.76 \\
\hline 1942 & 88.89 & 11.11 \\
\hline 1943 & 79.17 & 20.83 \\
\hline 1944 & 83.33 & 16.67 \\
\hline 1945 & 86.84 & 13.16 \\
\hline 1946 & 89.13 & 10.87 \\
\hline 1947 & 89.66 & 10.34 \\
\hline 1948 & 87.88 & 12.12 \\
\hline 1949 & 86.30 & 13.70 \\
\hline 1950 & 88.46 & 11.54 \\
\hline 1951 & 89.01 & 10.99 \\
\hline 1952 & 86.73 & 13.27 \\
\hline 1953 & 82.50 & 17.50 \\
\hline 1954 & 80.16 & 19.84 \\
\hline 1955 & 80.67 & 19.33 \\
\hline 1956 & 79.07 & 20.93 \\
\hline 1957 & 78.87 & 21.13 \\
\hline 1958 & 76.64 & 23.36 \\
\hline 1959 & 77.91 & 22.09 \\
\hline 1960 & 76.69 & 23.31 \\
\hline 1961 & 74.55 & 25.45 \\
\hline 1962 & 74.18 & 25.82 \\
\hline 1963 & 76.03 & 23.97 \\
\hline 1964 & 75.47 & 24.53 \\
\hline 1965 & 73.73 & 26.27 \\
\hline 1966 & 73.63 & 26.37 \\
\hline 1967 & 71.54 & 28.46 \\
\hline 1968 & 69.41 & 30.59 \\
\hline 1969 & 69.65 & 30.35 \\
\hline 1970 & 69.63 & 30.37 \\
\hline 1971 & 69.69 & 30.31 \\
\hline 1972 & 67.89 & 32.11 \\
\hline 1973 & 67.62 & 32.38 \\
\hline 1974 & 66.16 & 33.84 \\
\hline 1975 & 66.51 & 33.49 \\
\hline 1976 & 66.46 & 33.54 \\
\hline 1977 & 63.69 & 36.31 \\
\hline 1978 & 63.12 & 36.88 \\
\hline 1979 & 62.75 & 37.25 \\
\hline 1980 & 62.09 & 37.91 \\
\hline 1981 & 62.18 & 37.82 \\
\hline 1982 & 61.10 & 38.90 \\
\hline 1983 & 60.30 & 39.70 \\
\hline 1984 & 60.26 & 39.74 \\
\hline 1985 & 60.02 & 39.98 \\
\hline 1986 & 59.75 & 40.25 \\
\hline
\end{tabular}




\begin{tabular}{|c|c|c|}
\hline 1987 & 58.73 & 41.27 \\
\hline 1988 & 58.51 & 41.49 \\
\hline 1989 & 57.04 & 42.96 \\
\hline 1990 & 58.01 & 41.99 \\
\hline 1991 & 58.31 & 41.69 \\
\hline 1992 & 57.83 & 42.17 \\
\hline 1993 & 58.06 & 41.94 \\
\hline
\end{tabular}

Fuente: Jorge Gil-Mendieta, S. Schmidt, J. Castro y A. Ruiz. Base de Datos REDMEX_II, 2001. Laboratorio de Redes IIMAS-UNAM. 
Tabla 5. Gabinete de Miguel de la Madrid Hurtado Distribución de abogados y economistas

\begin{tabular}{|c|c|c|c|}
\hline Nombre & Abogados & Economistas & Otros \\
\hline MANUEL BARTLETT DIAZ & 1 & & \\
\hline BERNARDO SEPULVEDA AMOR & 1 & & \\
\hline JESUS SILVA HERZOG & & 1 & \\
\hline GUSTAVO PETRICIOLI ITURBIDE & & 1 & \\
\hline JUAN AREVALO GARDOQUI & & & 1 \\
\hline MIGUEL ANGEL GOMEZ ORTEGA & & & 1 \\
\hline EDUARDO PESQUEIRA OLEA & 1 & & \\
\hline RODOLFO FELIX VALDEZ & & & 1 \\
\hline DANIEL DIAZ DIAZ & & & 1 \\
\hline MARCELO J AVELLY GIRARD & 1 & & \\
\hline GUILLERMO CARRILLO ARENA & & & 1 \\
\hline MANUEL CAMACHO SOLIS & & 1 & \\
\hline $\begin{array}{l}\text { HECTOR HERNANDEZ } \\
\text { CERVANTES }\end{array}$ & & 1 & \\
\hline ARSENIO FARELL CUBILLAS & 1 & & \\
\hline LUIS MARTINEZ VILLICAÑA & & & 1 \\
\hline RAFAEL RODRIGUEZ BARRERA & 1 & & \\
\hline JESUS REYES HEROLES & & 1 & \\
\hline MIGUEL GONZALEZ AVELAR & 1 & & \\
\hline GUILLERMO SOBERON ACEVEDO & & & 1 \\
\hline FRANCISCO LABASTIDA OCHOA & & 1 & \\
\hline ALFREDO DEL MAZO GONZALEZ & & & 1 \\
\hline CARLOS SALINAS DE GORTARI & & 1 & \\
\hline PEDRO ASPE ARMELLA & & 1 & \\
\hline ANTONIO ENRIQUEZ SAVINAC & & & 1 \\
\hline FRANCISCO ROJAS GUTIERREZ & & & 1 \\
\hline IGNACIO PICHARDO PAGAZA & 1 & & \\
\hline PEDRO OJ EDA PAULLADA & 1 & & \\
\hline SERGIO GARCIA RAMIREZ & 1 & & \\
\hline RAMON AGUIRRE VELAZQUEZ & & & 1 \\
\hline $\begin{array}{l}\text { MIGUEL DE LA MADRID } \\
\text { HURTADO }\end{array}$ & 1 & & \\
\hline TOTAL & 11 & 8 & 11 \\
\hline PORCENTAJE & 36.66 & 26.66 & 36.66 \\
\hline
\end{tabular}

Fuente: J orge Gil-Mendieta, S. Schmidt, J. Castro y A. Ruiz. Base de Datos REDMEX_II, 2001. Laboratorio de Redes. IIMAS-UNAM 
Tabla 6. Gabinete de Carlos Salinas de Gortari Distribución de abogados y economistas

\begin{tabular}{|c|c|c|c|}
\hline Nombre & Abogados & Economistas & Otros \\
\hline MANUEL BARTLETT DIAZ & 1 & & \\
\hline JESUS SILVA HERZOG & & 1 & \\
\hline MANUEL CAMACHO SOLIS & & 1 & \\
\hline ARSENIO FARELL CUBILLAS & 1 & & \\
\hline CARLOS SALINAS DE GORTARI & & 1 & \\
\hline PEDRO ASPE ARMELLA & & 1 & \\
\hline FERNANDO GUTIERREZ BARRIOS & & & 1 \\
\hline $\begin{array}{l}\text { PATROCINIO GONZALEZ BLANCO } \\
\text { GARRIDO }\end{array}$ & 1 & & \\
\hline $\begin{array}{l}\text { JORGE CARPIZO MAC GREGOR } \\
\text { FERNANDO SOLANA MORALES }\end{array}$ & 1 & & 1 \\
\hline MANUEL TELLO MACIAS & & & 1 \\
\hline ANTONIO RUVIELLO BAZAN & & & 1 \\
\hline MAURICIO SCHLESKE SANCHEZ & & & 1 \\
\hline LUIS RUANO ANGULO & & & 1 \\
\hline JORGE DE LA VEGA DOMI NGUEZ & & 1 & \\
\hline CARLOS HANK GONZALEZ & & & 1 \\
\hline ANDRES CASO LOMBARDO & & 1 & \\
\hline EMILIO GAMBOA PATRON & & & 1 \\
\hline PATRICIO CHIRINOS CALERO & & 1 & \\
\hline LUIS DONALDO COLOSIO MURRIETA & & 1 & \\
\hline CARLOS ROJAS GUTIERREZ & & & 1 \\
\hline J AIME J OSE SERRA PUCHE & & 1 & \\
\hline VICTOR MANUEL CERVERA PACHECO & & & 1 \\
\hline ERNESTO ZEDILLO PONCE DE LEON & & 1 & \\
\hline J OSE ANGEL PESCADOR OSUNA & & 1 & \\
\hline JESUS KUMATE RODRIGUEZ & & & 1 \\
\hline FERNANDO HIRIART BALDERRAMA & & & 1 \\
\hline EMILIO LOZOYA THALMANN & & 1 & \\
\hline PEDRO J OAQUIN COLDWELL & 1 & & \\
\hline MARIA ELENA VAZQUEZ NAVA & & 1 & \\
\hline $\begin{array}{l}\text { MARIA DE LOS ANGELES MORENO } \\
\text { URIEGAS }\end{array}$ & & 1 & \\
\hline $\begin{array}{l}\text { GUI LLERMO J I MENEZ MORALES } \\
\end{array}$ & 1 & & \\
\hline ENRIQUE ALVAREZ DEL CASTILLO & 1 & & \\
\hline IGNACIO MORALES LECHUGA & 1 & & \\
\hline DIEGO VALADES RIOS & 1 & & \\
\hline HUMBERTO BENITEZ TREVIÑO & 1 & & \\
\hline MIGUEL MONTES GARCIA & 1 & & \\
\hline MANUEL AGUI LERA GOMEZ & & 1 & \\
\hline TOTAL & 11 & 15 & 12 \\
\hline PORCENTAJE & 28.94 & 39.47 & 31.57 \\
\hline
\end{tabular}


Fuente: Jorge Gil- Mendieta, S. Schmidt, J. Castro y A. Ruiz. Base de Datos REDMEX_II, 2001. Laboratorio de Redes. IIMAS-UNAM

Tabla 7. Gabinete de Ernesto Zedillo Ponce de León Distribución de abogados y economistas

\begin{tabular}{|c|c|c|c|}
\hline Nombre & Abogados & Economistas & Otros \\
\hline ARSENI O FARELL CUBILLAS & 1 & & \\
\hline JESUS REYES HEROLES & & 1 & \\
\hline FRANCISCO LABASTIDA OCHOA & & 1 & \\
\hline IGNACIO PICHARDO PAGAZA & 1 & & \\
\hline CARLOS ROJ AS GUTIERREZ & & & 1 \\
\hline JIME J OSE SERRA PUCHE & & 1 & \\
\hline ERNESTO ZEDILLO PONCE DE LEON & & 1 & \\
\hline ESTEBAN MOCTEZUMA BARRAGÁN & 1 & 1 & \\
\hline $\begin{array}{l}\text { EMILIO CHUAYFFET CHEMOR } \\
\text { DIODORO CARRASCO ALTAMIRANO }\end{array}$ & 1 & 1 & \\
\hline $\begin{array}{l}\text { HERMINIO BLANCO MENDOZA } \\
\text { HERMINIO BLANCO MENDOZA }\end{array}$ & & 1 & \\
\hline ENRIQUE CERVANTES AGUIRRE & & & 1 \\
\hline ARTURO WARMAN GRYJ & & & 1 \\
\hline OSCAR ESPINOSA VI LLARREAL & & & 1 \\
\hline J OSÉ ÁNGEL GURRIA TREVIÑO & & 1 & \\
\hline GUILLERMO ORTIZ MARTÍNEZ & & 1 & \\
\hline FAUSTO ALZATI ARAIZA & 1 & 1 & \\
\hline RUBÉN VALDÉZ ABASCAL & 1 & & \\
\hline JOSÉ RAMÓN LORENZO FRANCO & & & 1 \\
\hline CARLOS RUIZ SACRISTÁN & & & 1 \\
\hline CARLOS JARQUE URIBE & & 1 & \\
\hline MIGUEL LIMÓN ROJAS & 1 & & \\
\hline NORMA SAMANIEGO & & 1 & \\
\hline LUIS TELLEZ KUENZLER & 1 & 1 & \\
\hline $\begin{array}{l}\text { SANTIAGO OÑATE LABORDE } \\
\text { JESUS JAVIER BONILLA GARCIA }\end{array}$ & 1 & 1 & \\
\hline $\begin{array}{l}\text { J OSÉ ANTONI O GONZÁLEZ } \\
\text { FERNÁNDEZ }\end{array}$ & 1 & & \\
\hline SILVIA HERNÁNDEZ & & & 1 \\
\hline TOTAL & 10 & 14 & 7 \\
\hline PORCENTAJE & 32.25 & 45.16 & 22.58 \\
\hline
\end{tabular}

Fuente: Jorge Gil-Mendieta, S. Schmidt, J. Castro y A. Ruiz. Base de Datos REDMEX_II, 2001

Laboratorio de Redes. IIMAS-UNAM 
Figure 1. Public Functionaries with Law or Economy Studies and Others 1940-1993 (Complete data base)

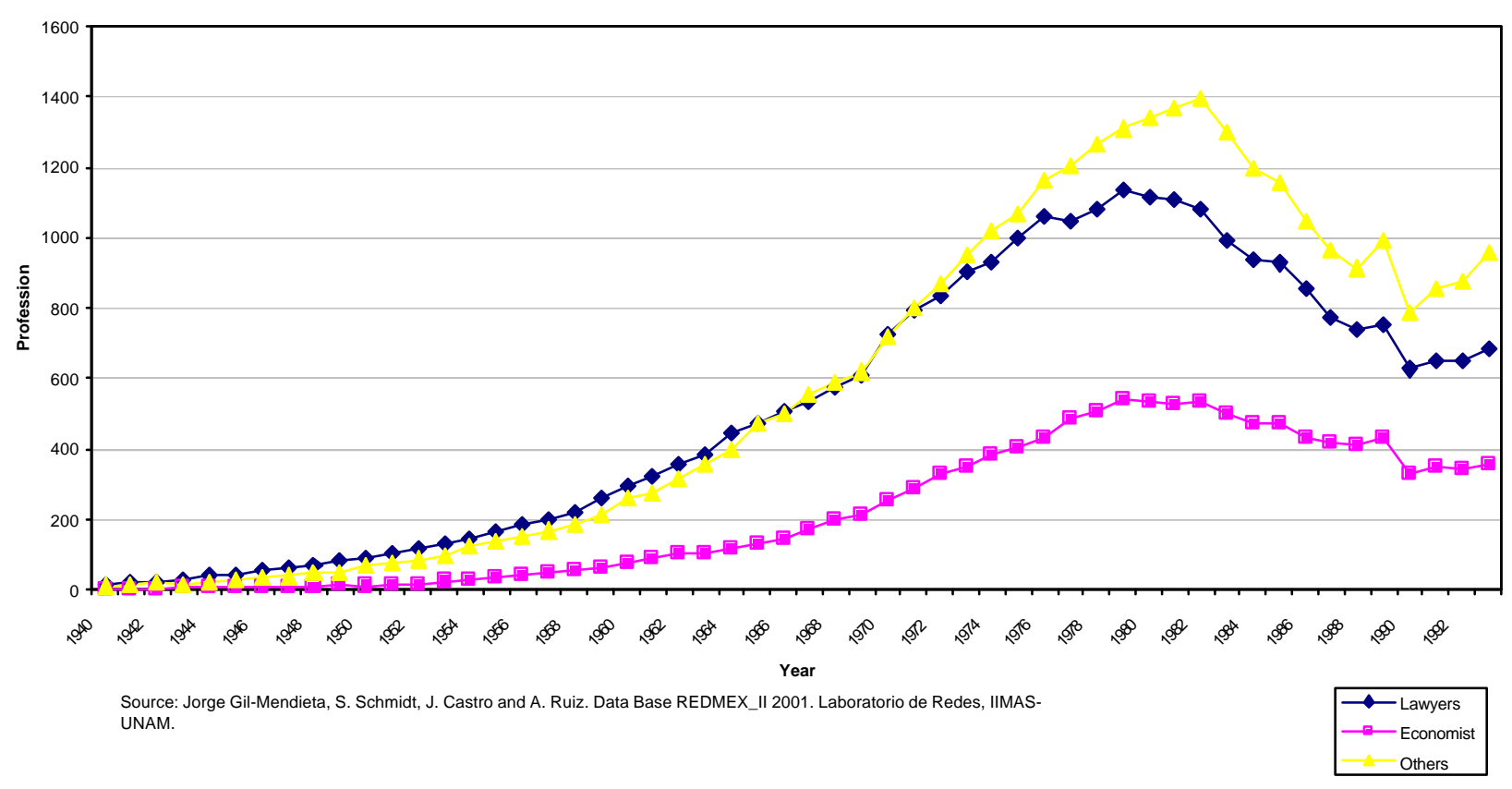


Figure 2. Lawyers and Economist 1940-1993 (Complete data base)

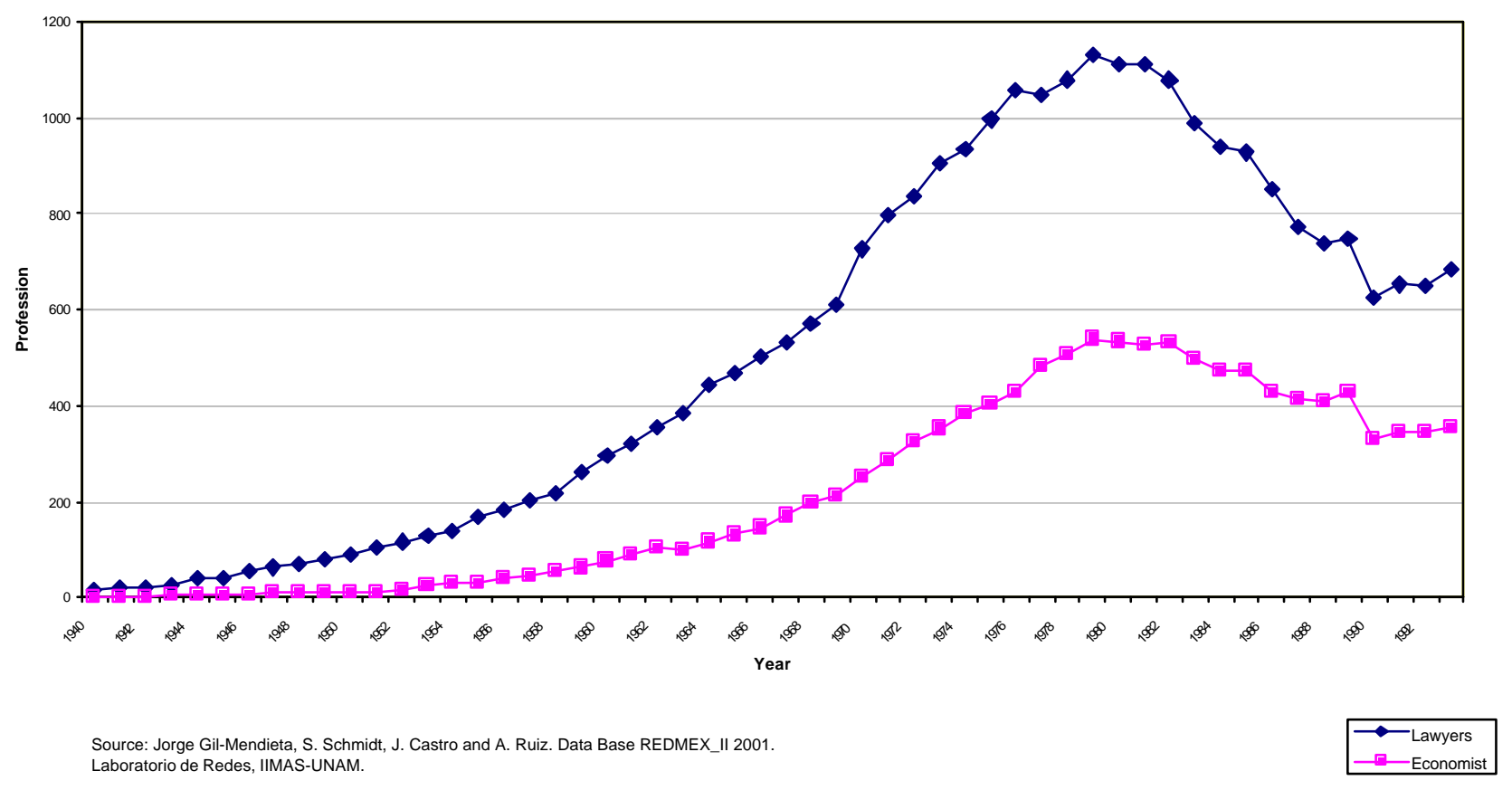


Figure 3. Percentage as Part of the Total of Economist Plus Lawyers 1940-1993.

(Complete data base)

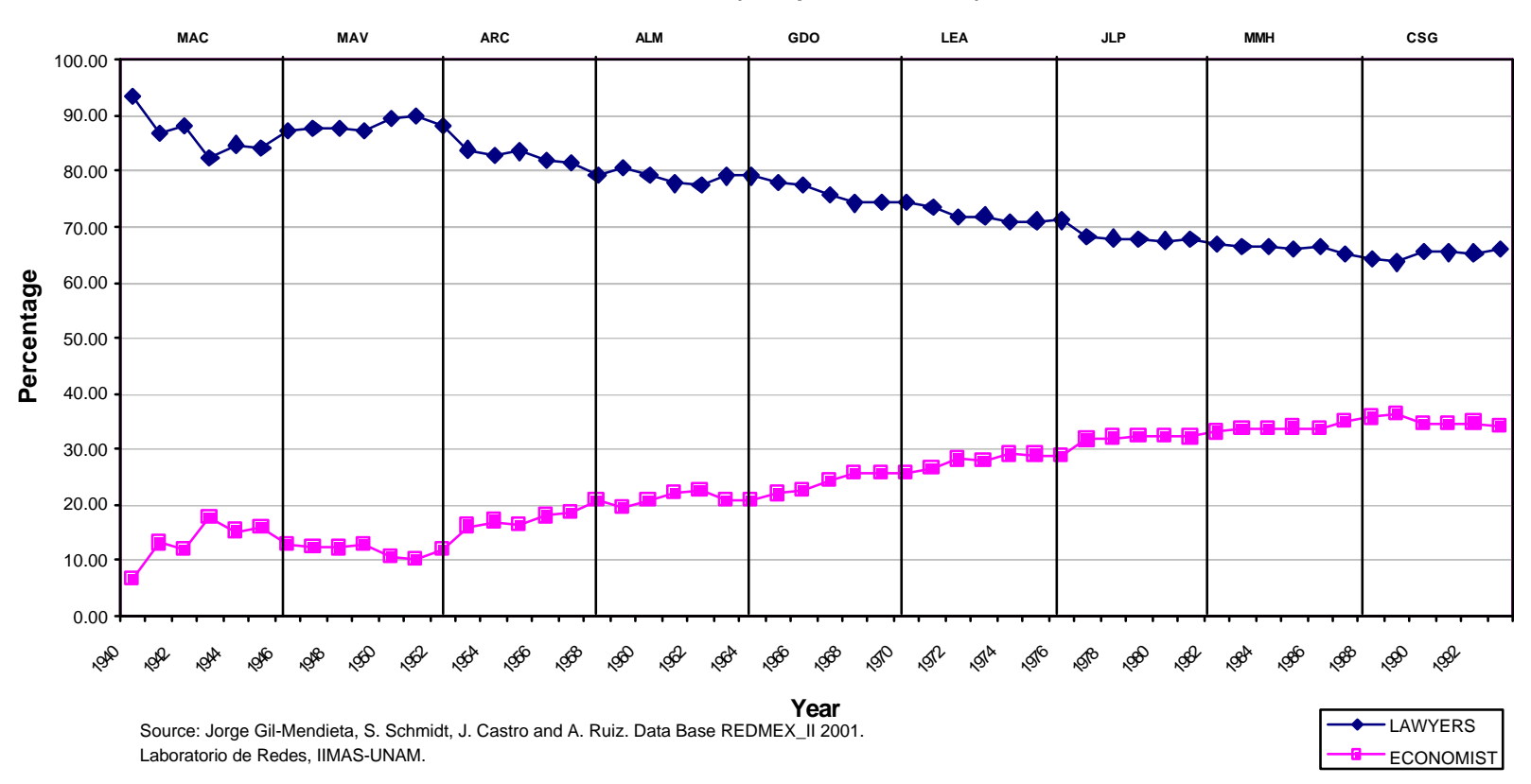




$$
\text { N }
$$


Figure 5. Lawyers and Economist 1940-1993

That where related to the 85 people at the De La Madrid, Salinas and Zedillo cabinets

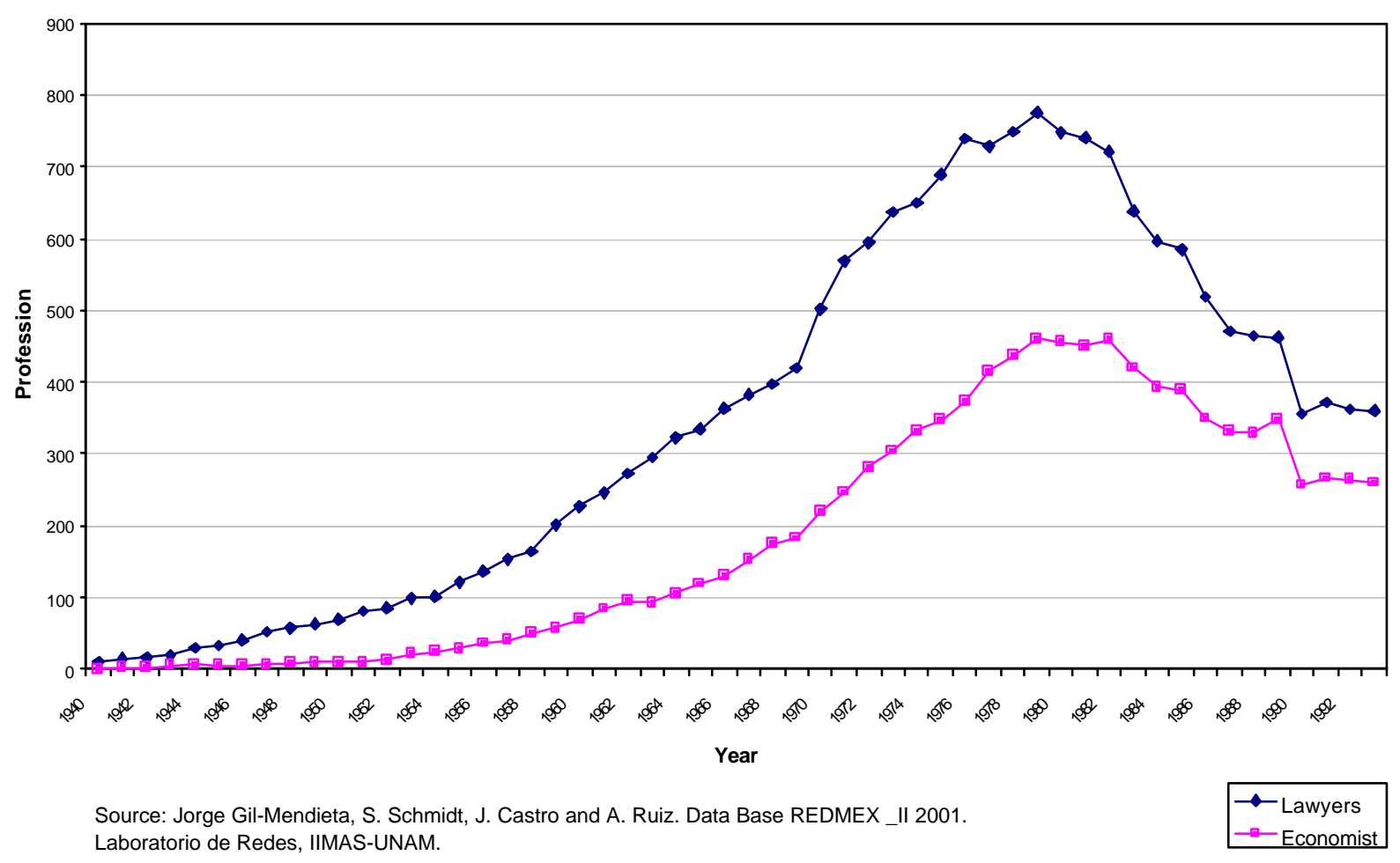


Figure 6. Public Functionaries with Law or Economy Studies 1940-1993

That where related to the 85 people at the De La Madrid, Salinas and Zedillo cabinets

(Percentage as part of the total economist plus lawyers)

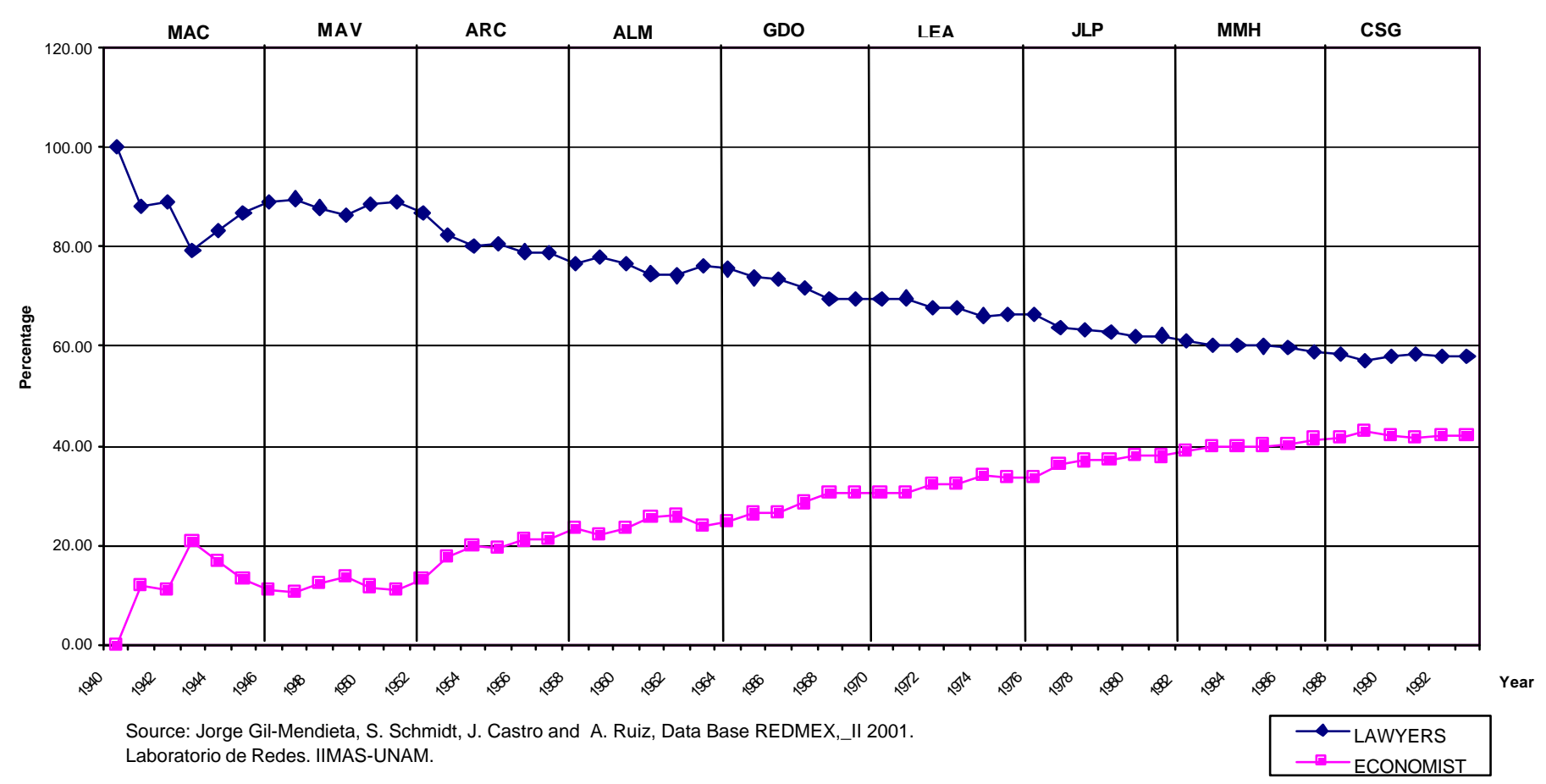


